\title{
Kinematics Analysis of Multi-link Mechanism for Miniature Servo Press Based on Matlab
}

\author{
Xinjian Lu ${ }^{1,2,3}$ \\ ${ }^{1}$ School of Mechanical Engineering, Nanjing Institute of Technology, Nanjing 211167, China \\ ${ }^{2}$ Research Department of Intelligent Manufacturing Equipment, Nanjing Institute of Technology, Nanjing 211167, China \\ ${ }^{3}$ Yangzhou SHUANG YING Metal Forming Machine Co., Ltd. Yangzhou 225000, China
}

\begin{abstract}
The degree of freedom for a multi-link drive mechanism of a 50kN miniature servo press was calculated. The kinematics equations of the mechanism was established based on the rod group method. The choice or design basis for rod length and some hinge points were proposed. The multi-linkage mechanism's line segment model was constructed in SolidWorks and a set of design parameters was obtained that met the requirements. The $\mathrm{M}$ file of kinematic equations were wrote and calculated in Matlab and the displacement, velocity and acceleration curve of the slider were obtained. Compared with the crank-slider mechanism, the mechanism has the features of quick return, small return speed, slightly smaller speed and larger acceleration near B.D.C. Therefore, the speed and acceleration characteristics of the mechanism were inferior to the crank-slider mechanism in the case of reverse rotation and higher speed.
\end{abstract}

\section{Introduction}

Multi-link drive mechanism is widely used in mechanicalpresses. For example, the presses with eight-link (1) or the six-link (double-action press) (2) were mainly used for the body cover's stamping for automobiles, the presses with double crank mechanism (3) mechanism were mainly used for stamping parts of home appliances and auto parts industry, mainlymulti-link drive systems for micro-motor and microelectronic stamping industry are: (4)symmetrical toggle lever(Kyori and Fair Oaks adopted),(5)double-link (Yamada Dobby adopted) and (5)leverage(Bruderer adopted) and so on. By using multi-link mechanism the contact speed and acceleration near bottom dead center(B.D.C) can be reduced in punching, and the life of molds can be improved also. There are few researches on the driving mechanism of mini press (the nominal force is usually less than $100 \mathrm{kN}$ )and crank-slider mechanism was usually used as working mechanism. With the popularity of servo drive technology, more and more servo drives are used in large presses. The multi-link drive's using in a mini presswill help to reduce the torque required during the blanking process, reduce the servo motor's power and increase the flexibility of the press. In this paper, a $50 \mathrm{kN}$ micro servo press (for precision electronic connector stamping) drive system has been studied. It's kinematic equations were derived and the slider displacement, velocity and acceleration curve are calculated based on Matlab. and curve shape's influencing factors were studied also. Research results for reference only.

\section{Multi-link drive mechanism}

The 50kN mini servo press multi-link drive mechanism was shown in Figure 1. The mechanism is composed of crank rocker mechanism rocker slider mechanism in series. By rational design the mechanism can save torque(6).That is to say, the same torque can produce greater stamping force. The freedom of this body is:

$$
f=3 \times 5-2 \times 7=1
$$

Therefore, the mechanism has a definite movement. The movement of the mechanism is as follows: The crank 2 rotates around the center $\mathrm{O}$ of the coordinate system and is hinged with the connecting rod 3 to the point $\mathrm{P}_{23}$, the connecting rod 3 drives the connecting rod 4 to swing within a certain angle range, the inner part of the connecting rod 4 and the frame are hinged at point $\mathrm{P}_{14}$, one end of the connecting rod 4 is hinged with the connecting rod 3 at point $\mathrm{P}_{34}$ and the other end is hinged with the connecting rod 5 at point $\mathrm{P}_{45}$, the slider 6 reciprocates up and down in the rail of the rack 1 , and is hinged with the connecting rod 5 through the hinge point $P_{56}$.

\footnotetext{
${ }^{\mathrm{a}}$ Corresponding author: jw41281997@ tom.com
} 


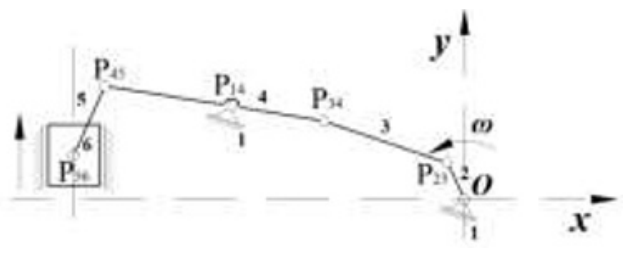

Figure 1. Multi-link drive mechanism

\section{Kinematics equations}

The coordinate system of the multi-link drive mechanism was shown in Figure 1. The origin of the coordinates is located at the center of rotation $\mathrm{O}$ of the crank 2, with the $\mathrm{X}$-axis horizontal to the right and the $y$-axis vertically upwards. Kinematic equations of this mechanism were established by using rod group analysis. The mechanism is broken down into one side link and two II grade rod groups as shown in Figure 2. The location equations of each hinge point were established. Speed and acceleration for hinge points were obtained by calculating the first and second derivatives of the position equation over time.

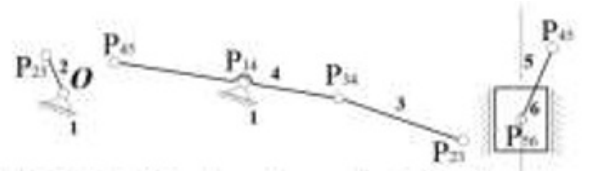

Side link Grade rod group 1 Grade rod group 2

Figure 2. Side link and II grade rod groups

\subsection{Side link}

As shown in Figure 3, the radius of the crank 2 is $r$, the rotation angle is $\varphi$, the angular velocity is $\omega$, and the displacement of the hinge point $\mathrm{P}_{23}$ between the crank 2 and the connecting rod is:

$$
\left.\begin{array}{l}
x_{23}=r \cos \varphi \\
y_{23}=r \sin \varphi
\end{array}\right\}
$$

By calculating the first and second derivatives of equation (2) over time the velocity and acceleration of the hinge point $\mathrm{P}_{23}$ were obtained (irrespective of the fluctuation of the crank rotation speed during punching, and the angular acceleration of the crank when the machine was started and braked).

$$
\left.\begin{array}{c}
\dot{x}_{23}=-\omega r \sin \varphi \\
\dot{y}_{23}=\omega r \cos \varphi
\end{array}\right\}
$$

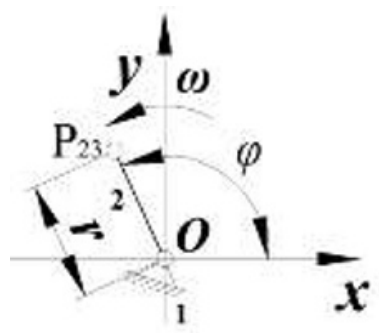

Figure 3. Side link

\subsection{Grade rod group 1}

Rod group 1 was shown in Figure 4. The length of the connecting rod 3 was set to be $l_{3}$. The $x$ coordinate and the $y$ coordinate of the hinge point $\mathrm{P}_{14}$ were respectively $x_{14}$ and $y_{14}$, which were known quantities. The distance between the hinge points $\mathrm{P}_{34}$ and $\mathrm{P}_{45}$ was $l_{4}$, and the distance between the hinge points $\mathrm{P}_{34}$ and $\mathrm{P}_{14}$ was $l_{41}$.

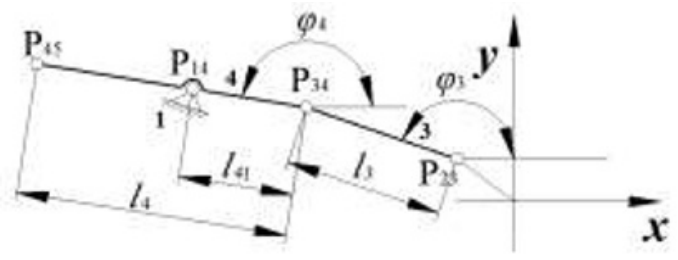

Figure 4. II grade rod group 1

The displacement equations for hinge point $\mathrm{P}_{34}$ between the connecting rod 3 and the connecting rod 4 was:

$$
\left.\begin{array}{l}
x_{34}=x_{23}+l_{3} \cos \varphi_{3} \\
y_{34}=y_{23}+l_{3} \sin \varphi_{3}
\end{array}\right\}
$$

By calculating the first and second derivatives of equation (5) over time the velocity and acceleration of the hinge point $\mathrm{P}_{34}$ were obtained.

$$
\begin{aligned}
& \dot{x}_{34}=\dot{x}_{23}-\dot{\varphi}_{3} l_{3} \sin \varphi_{3} \\
& \left.\dot{y}_{34}=\dot{y}_{23}+\dot{\varphi}_{3} l_{3} \cos \varphi_{3}\right\} \\
& \left.\ddot{x}_{34}=\ddot{x}_{23}-l_{3}\left(\dot{\varphi}_{3}^{2} \cos \varphi_{3}+\ddot{\varphi}_{3} \sin \varphi_{3}\right)\right\} \\
& \left.\ddot{y}_{34}=\ddot{y}_{23}+l_{3}\left(\ddot{\varphi}_{3} \cos \varphi_{3}-\dot{\varphi}_{3}^{2} \sin \varphi_{3}\right)\right\}
\end{aligned}
$$

The displacement equation of the hinge point $\mathrm{P}_{14}$ between the connecting rod 4 and the body 1 was:

$$
\left.\begin{array}{l}
x_{41}=x_{34}+l_{41} \cos \varphi_{4} \\
y_{41}=y_{34}+l_{41} \sin \varphi_{4}
\end{array}\right\}
$$

The velocity of the hinge point $\mathrm{P}_{14}$ was obtained by calculating the first derivation of equation (8) over time. Since the hinge point is a fixed point, the speed value should be zero.

$$
\left.\begin{array}{l}
0=\dot{x}_{34}-\dot{\varphi}_{4} l_{41} \sin \varphi_{4} \\
0=\dot{y}_{34}+\dot{\varphi}_{4} l_{41} \cos \varphi_{4}
\end{array}\right\}
$$

Solving Equations (5) and (8).

$$
\begin{aligned}
& \varphi_{4}=2 \pi+2 \operatorname{arctg} \frac{B-\sqrt{B^{2}+A^{2}-C^{2}}}{A+C} \\
& \varphi_{3}=\operatorname{atan} 2\left(y_{34}, x_{34}\right)
\end{aligned}
$$

In the above formula,

$$
\begin{gathered}
A=x_{41}-\mathrm{rcos} \varphi \\
B=y_{41}-\mathrm{rsin} \varphi \\
C=-\frac{l_{3}^{2}-l_{41}^{2}-\mathrm{r}^{2}-x_{41}^{2}-y_{41}^{2}+2\left(x_{41} \mathrm{r} \cos \varphi+y_{41} \mathrm{r} \sin \varphi\right)}{2 l_{41}}
\end{gathered}
$$

The angular velocity and angular acceleration for $\varphi_{4}$ were:

$$
\dot{\varphi}_{4}=\frac{\dot{\mathrm{C}}-\dot{\mathrm{A}} \cos \varphi_{4}-\dot{\mathrm{B}} \sin \varphi_{4}}{\mathrm{~B} \cos \varphi_{4}-\mathrm{A} \sin \varphi_{4}}
$$




$$
\begin{aligned}
& \ddot{\varphi}_{4} \\
& =\frac{\ddot{C}-\ddot{A} \cos \varphi_{4}+\dot{A} \sin \varphi_{4} \dot{\varphi}_{4}-\ddot{B} \sin \varphi_{4}-\dot{B} \cos \varphi_{4} \dot{\varphi}_{4}}{B \cos \varphi_{4}-A \sin \varphi_{4}} \\
& -\frac{\left(\dot{C}-\dot{A} \cos \varphi_{4}-\dot{B} \sin \varphi_{4}\right)\left(\dot{B} \cos \varphi_{4}-\dot{A} \sin \varphi_{4}\right)}{\left(B \cos \varphi_{4}-A \sin \varphi_{4}\right)^{2}} \\
& +\frac{\left(\dot{C}-\dot{A} \cos \varphi_{4}-\dot{B} \sin \varphi_{4}\right)\left(B \sin \varphi_{4}+A \cos \varphi_{4}\right) \dot{\varphi}_{4}}{\left(B \cos \varphi_{4}-A \sin \varphi_{4}\right)^{2}}
\end{aligned}
$$

In the formula(13),

$$
\begin{array}{cc}
\dot{A}=\omega \mathrm{rsin} \varphi & \ddot{A}=\omega^{2} \mathrm{r} \cos \varphi \\
\dot{B}=-\omega \mathrm{rcos} \varphi & \dot{B}=\omega^{2} \mathrm{r} \sin \varphi \\
\dot{C}=\frac{\omega r\left(y_{41} \cos \varphi-x_{41} \sin \varphi\right)}{\mathrm{l}_{41}} \\
\ddot{C}=\frac{-\omega^{2} \mathrm{r}\left(y_{41} \sin \varphi+x_{41} \cos \varphi\right)}{\mathrm{l}_{41}}
\end{array}
$$

The angular velocity and angular acceleration for $\varphi_{3}$ were:

$$
\begin{aligned}
& \dot{\varphi}_{3}=-\frac{\omega \mathrm{r} \sin \varphi+1_{41} \sin \varphi_{4} \dot{\varphi}_{4}}{\mathrm{l}_{3} \sin \varphi_{3}} \\
& \ddot{\varphi}_{3}=\frac{\left(\omega \mathrm{rsin} \varphi+l_{41} \sin \varphi_{4} \dot{\varphi}_{4}\right) \cos \varphi_{3} \dot{\varphi}_{3}}{l_{3}\left(\sin \varphi_{3}\right)^{2}} \\
& -\frac{\omega^{2} \mathrm{r} \cos \varphi+l_{41}\left(\cos \varphi_{4} \dot{\varphi}_{4}^{2}+\sin \varphi_{4} \ddot{\varphi}_{4}\right)}{l_{3} \sin \varphi_{3}}
\end{aligned}
$$

The displacement equation of the hinge point $\mathrm{P}_{45}$ between the connecting rod 4 and the connecting rod 5 was:

$$
\left.\begin{array}{c}
x_{45}=x_{34}+l_{4} \cos \varphi_{4} \\
y_{45}=y_{34}+l_{4} \sin \varphi_{4}
\end{array}\right\}
$$

The speed and acceleration of the hinge point $\mathrm{P}_{45}$ were obtained by calculating the first and second derivatives of equation (16) over time.

$$
\left.\left.\begin{array}{l}
\dot{x}_{45}=\dot{x}_{34}-l_{4} \sin \varphi_{4} \dot{\varphi}_{4} \\
\dot{y}_{45}=\dot{y}_{34}+l_{4} \cos \varphi_{4} \dot{\varphi}_{4}
\end{array}\right\} \begin{array}{l}
\ddot{x}_{45}=\ddot{x}_{34}-l_{4}\left(\sin \varphi_{4} \ddot{\varphi}_{4}+\cos \varphi_{4} \dot{\varphi}_{4}^{2}\right) \\
\ddot{y}_{45}=\ddot{y}_{34}+l_{4}\left(\cos \varphi_{4} \ddot{\varphi}_{4}-\sin \varphi_{4} \dot{\varphi}_{4}^{2}\right)
\end{array}\right\}
$$

\subsection{Grade rod group 2}

Rod group 2 was shown in Figure 5. The length of the connecting rod 5 was set to be $l_{5}$, and the $x$ coordinate $x_{56}$ of the hinge point $\mathrm{P}_{56}$ was a known quantity. The $y$ coordinate $\left(y_{56}\right)$ of the hinge point P56 was the displacement $s$ for the slider 6 . The displacement equation of hinge point $\mathrm{P}_{56}$ was:

$$
\left.\begin{array}{l}
x_{56}=x_{45}+l_{5} \cos \varphi_{5} \\
y_{56}=y_{45}+l_{5} \sin \varphi_{5}
\end{array}\right\}
$$

The result of solving was:

$$
\varphi_{5}=2 \pi-2 \operatorname{arctg} \sqrt{\frac{1-D}{1+D}}
$$

In the formula(20),

$$
D=\frac{x_{56}-x_{45}}{l_{5}}
$$

The angular velocity and angular acceleration for $\varphi_{5}$ were:

$$
\dot{\varphi}_{5}=\frac{\dot{x}_{45}}{l_{5} \sin \varphi_{5}}
$$

$$
\ddot{\varphi}_{5}=\frac{\ddot{x}_{45}}{l_{5} \sin \varphi_{5}}-\frac{\dot{x}_{45} \cos \varphi_{5} \dot{\varphi}_{5}}{l_{5}\left(\sin \varphi_{5}\right)^{2}}
$$

Therefore, the speed and acceleration of the slider 6 were respectively:

$$
\begin{aligned}
& v=\dot{y}_{56}=\dot{y}_{45}+l_{5} \cos \varphi_{5} \dot{\varphi}_{5} \\
& \alpha=\ddot{y}_{56}=\ddot{y}_{45}+l_{5}\left(\cos \varphi_{5} \ddot{\varphi}_{5}-\sin \varphi_{5} \dot{\varphi}_{5}^{2}\right)
\end{aligned}
$$

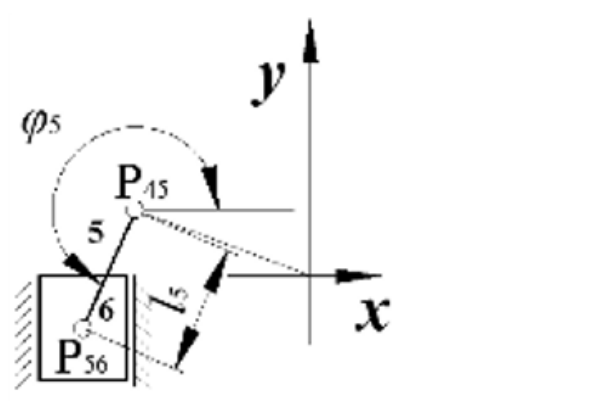

Figure 5. II grade rod group 2

\section{Determination of rod design parameters}

The main design parameters of the $50 \mathrm{kN}$ mini servo press were shown in Table 1:

Table 1. The main design parameters.

\begin{tabular}{|c|c|c|}
\hline item & value & unit \\
\hline Nominal force & 50 & $\mathrm{kN}$ \\
\hline Stroke length(max) & 40 & $\mathrm{~mm}$ \\
\hline Activating point & 1.4 & $\mathrm{~mm}$ \\
\hline No. of strokes(max) & 150 & \\
\hline Open height & 300 & $\mathrm{~mm}$ \\
\hline $\begin{array}{c}\text { Overall Dimensions } \\
\text { (L.R. xH x F.B.) }\end{array}$ & $700 \times 1000 \times 1000$ & $\mathrm{~mm}$ \\
\hline
\end{tabular}

\subsection{Design guidelines for link system}

1. Taking into account the design and manufacturing factors the design value of the crank radius $r$ is within $50 \mathrm{~mm}$;

2. According to my past experience, the pin diameter of the rod system is about $30 \mathrm{~mm}$. Considering the wall thickness of the pin hole and the movement space of each rod, the length of each rod or part is about $80 \mathrm{~mm}$;

3 . The connecting rod coefficient $\left(r / l_{2}\right)$ should be designed within $0.1 \sim 0.2$ (7),for high-speed and fast presses will be less than 0.1 ; 
4. The crank-rocker mechanism should meet the conditions of the rod length and the existence of the crank (8);

5. In order to avoid the dead point of the mechanism due to friction, the angle between the connecting rod 5 and the vertical line should be designed within $30^{\circ}$;

6. The overall length of the rod system should be less than the overall dimensions of the front and back directions.

\subsection{Rod parameters}

In the SolidWorks software, the origin was taken as the crank center point $\mathrm{O}$ of the mechanism, the connecting rods were replaced by line segments, the point drawn in the line segment was considered as the hinge point in the connecting rod, and the multi-link mechanism can be obtained. The length of the rods and the coordinates of the hinges were given appropriate values to ensure that the mechanism can operate without dead point when the mouse dragging the mechanism's crank. Based on MATLAB, the kinematics analysis program of the mechanism was written in the form of $\mathrm{M}$ file, and then the parameters of the multilink system were adjusted slightly. At the same time, the kinematics equations were corrected (confirming the quadrant where the angle was), and the rod parameters that satisfy the design requirements were shown in Table II

Table 2. Rod length and position parameters( $\mathrm{mm})$.

\begin{tabular}{|c|c|c|c|c|c|c|c|c|}
\hline Item & $\mathrm{r}$ & $l_{3}$ & $l_{41}$ & $l_{4}$ & $l_{5}$ & $x_{14}$ & $y_{14}$ & $x_{56}$ \\
\hline Value & 14.58 & 330 & 80 & 184 & 100 & -380 & 60 & -465 \\
\hline
\end{tabular}

\section{Calculation results}

By moving the axes, it was guaranteed that the curve changes from top dead center $\left(0^{\circ}\right)$ as the crank angle changes. For comparison, the slider displacement, velocity and acceleration of the crank-slider mechanism ( slider stroke: $40 \mathrm{~mm}$, crank radius : $20 \mathrm{~mm}$, link length : $300 \mathrm{~mm}$ ) were given in the figures below.

Slider displacement curves were shown in Figure 6. Compared with the crank-slider mechanism, the position of the B.D.C of the slider for the multi-link mechanism was slightly shifted to the left by about $3^{\circ}$ due to the connected crank-rocker'quick-return characteristics. And the mechanismwas able to reach capacity early.

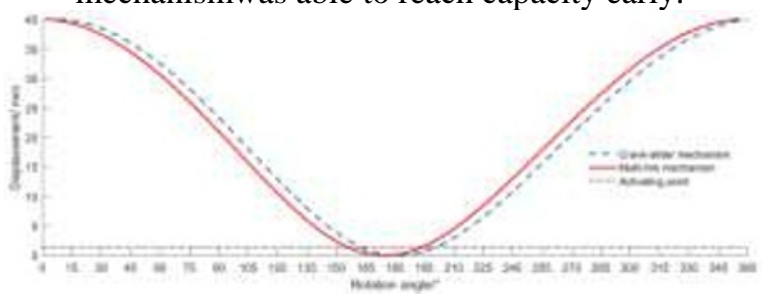

Figure 6. Slider displacement curves

Slider velocity curves were shown in Figure 7. Compared with the crank-slider mechanism, The multi-link mechanism's return speed of $292.85 \mathrm{~mm} / \mathrm{s}$ was slightly less than the crank- slider mechanism's $314.85 \mathrm{~mm} / \mathrm{s}$. Near B.D.C (left side of the curves), the speed of the multi-link mechanism was slightly less than the crank-slider mechanism, and it was help to precision punching. When leaving B.D.C(right side of the curves), the multi-link mechanism's speed was larger than crank-slider mechanism's.

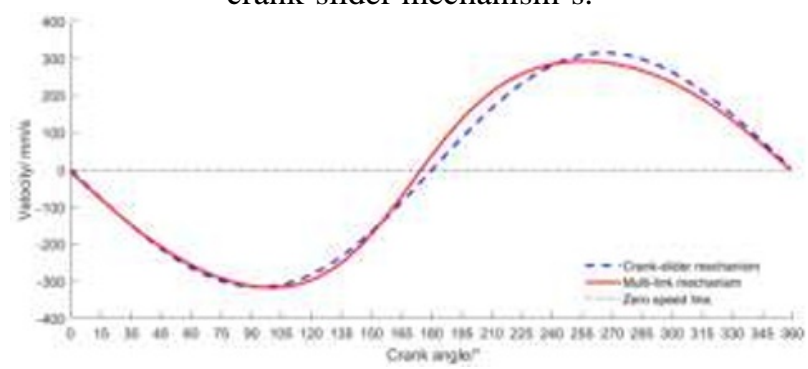

Figure 7. Slider velocity curves

Slider velocity curves were shown in Figure VIII. Compared with the crank-slider mechanism, the acceleration of the multi-link mechanism near B.D.C reaches $6650.1 \mathrm{~mm} / \mathrm{s}^{2}$, which is 1.26 times the $5263.8 \mathrm{~mm} / \mathrm{s}^{2}$ of the crank-slider mechanism.

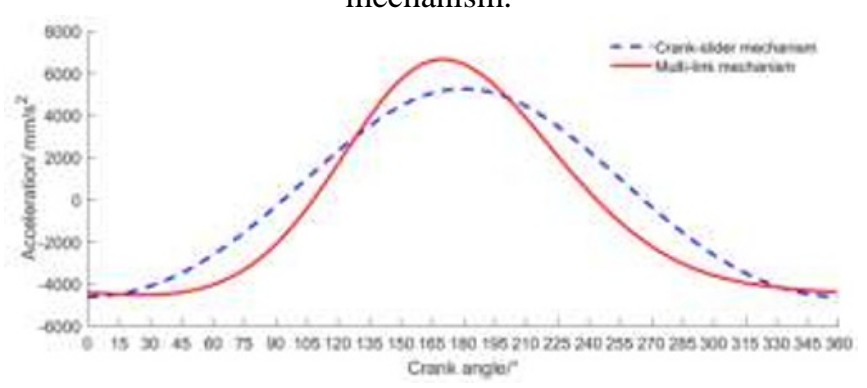

Figure 8. Slider acceleration curves

\section{Conclusions}

The kinematic equations of a multi-link drive mechanism for the $50 \mathrm{kN}$ servo press were established, and the design principles of the rod-system parameters were put forward. A set of parameters were given to meet the design requirements. The crank radius of this mechanism was only $72.9 \%$ of the crank radius in the crank-slider mechanism at the same slider stroke. Kinematics equations were established in Matlab and calculated. Compared with the crank-slider mechanism, the mechanism has the features of quick return, small return speed, slightly smaller speed and larger acceleration near B.D.C. Therefore, the speed and acceleration characteristics of the mechanism were inferior to the crank-slider mechanism in the case of reverse rotation and higher speed.

\section{Acknowledgements}

The author wishes to thank the Opening Project of Research Department of Intelligent Manufacturing Equipment of Nanjing Institute of Technology(project number: PTKJ201608) and The introduction of talent research fund project of Nanjing Institute of Technology(project number:YKJ201503).

\section{References}

1. Lian Xia,Jin Zhang, Jiang Han, Yunshan Wang and Liang HE. Kinematics analysis of eight-link mechanical 
press[J],Forging\&Stamping Technology,2012,37(4):158161(in Chinese).

2. Guiwei Dong,Yiguo Luan,Linlin Sun and Yanjun Yu. Kinematic analysis and Optimum Design for Multi-link Mechanism in Drawing Press[J]. China Metalforming Equipment \& Manufacturing Technology,2010,41(4):2730.(in Chinese).

3. Yujian Xiang,Shisong Tang,Zhiwei Zhou,Kuan She and Zhi Liu.Optimal Design of Rod System for Double Crank Servo Press on the Basis of Genetic Algorithm[J]. China Metalforming Equipment \& Manufacturing Technology,2017,52(3):17-19.(in Chinese).

4. Cankun He. Kinematic Characteristics Analysis of Multilink High-speed Press $[\mathrm{J}]$. China Metalforming Equipment\&ManufacturingTechnology,2013,48(5):1921.(in Chinese).

5. Xinjian Lu,Zunmang Ke, Sihong Zhu and Jun Zhu. Research on Bottom Dead Center (B.D.C) of High Speed Precision Press $[\mathrm{J}]$. Forging\&Stamping Technology,2010,35(1):87-92(in Chinese).

6. Meilin Zhang, Youchen Zhang and Liyan Zhang. Mechanical Innovation Design (2nd Edition)[M]. Beijing: Chemical Industry Press, 2010.(in Chinese)

7. Deyu He. Crank Press[M]. Beijing: China Machine Press, 1981.(in Chinese)

8. Yu Zhu. Fundamentals of Mechanical Design[M]. Beijing: Peking University Press, 2013.(in Chinese) 